\title{
Drug Persistence of Biologic Treatments in Psoriasis: A Swedish National Population Study
}

\author{
Marcus Schmitt-Egenolf (1) - Jonatan Freilich - Natalia M. Stelmaszuk-Zadykowicz • \\ Eydna Apol · Jes B. Hansen · Lars-Åke Levin
}

Received: June 24, 2021 / Accepted: September 15, 2021

(C) The Author(s) 2021

\begin{abstract}
Introduction: Biologic treatments for psoriasis are commonly switched. Treatment persistence represents an important parameter related to long-term therapeutic performance. The objective of the study was to analyse the real-world persistence with biologics over time in the treatment of psoriasis.
\end{abstract}

Methods: A retrospective observational study of adults with psoriasis was conducted based on Swedish national registry data from 2010 to

Supplementary Information The online version contains supplementary material available at https:// doi.org/10.1007/s13555-021-00616-7.

M. Schmitt-Egenolf $(\bowtie) \cdot$ J. Freilich

Department of Public Health and Clinical Medicine, Dermatology, Umeå University, SE-901 85 Umeå, Sweden

e-mail: marcus.schmitt-egenolf@umu.se

URL: http://www.derma.org

M. Schmitt-Egenolf

Centre for Pharmacoepidemiology, Karolinska

Institutet, Stockholm, Sweden

J. Freilich · N. M. Stelmaszuk-Zadykowicz

Parexel International, Stockholm, Sweden

E. Apol · J. B. Hansen

LEO Pharma A/S, Ballerup, Denmark

L.-A. Levin

Department of Health, Medicine and Caring

Sciences (HMV), Linköping University, Linköping,

Sweden
2018. Patients included were treated with a biologic between 2010 and 2018. Treatment episodes were identified from the drug's date of dispensation recorded in the Prescribed Drug Register to the end of supply of the drug. Median persistence was estimated by Kaplan-Meier survival curves for patients who received adalimumab, etanercept, secukinumab, ustekinumab and ixekizumab. Descriptive analysis of change in persistence over time for 3-year running cohorts was also carried out.

Results: A total of 2292 patients were analysed. Patients who received ustekinumab had the longest median persistence [ 49.3 months, 95\% confidence interval (CI) 38.0-59.1] and etanercept the shortest (16.3 months, 95\% CI 14.5-19.0). Median persistence was longer in biologic-naive than biologic-exposed patients. Persistence for ustekinumab decreased by almost $50 \%$ over the study period, from a median of 62.3 (95\% CI $45.6-\infty)$ months in 2010-2011 to 32.7 (21.2-49.3) months in 2014-2016.

Conclusions: Persistence with biologics was, on average, relatively low, given the chronic nature of psoriasis. Changes in persistence over time seemed to be attributable to changes in the therapeutic landscape, providing patients with more options to switch biologic treatments if their current management was considered suboptimal. 
Keywords: Biologics; Persistence; Psoriasis; Real-world data

\section{Key Summary Points}

\section{Why carry out this study?}

Persistence for biologic therapy in the treatment of moderate-to-severe psoriasis is unsatisfactory.

This patient-level registry study from Sweden characterized the persistence with individual biologics and the changes in persistence over time.

\section{What was learned from the study?}

The results of this study may aid in clinical decision-making when choosing a biological therapy for patients with psoriasis by contributing important evidence on the differential persistence over time for each biologic to the body of evidence on persistence of biologic therapy, which usually only focusses on persistence at a given point in time.

The findings may inform clinical decisionmaking based on evidence on the differential persistence over time for each biologic.

\section{INTRODUCTION}

Biologic therapies with diverse mechanisms of action have been developed to treat moderateto-severe psoriasis. Targets for biologics are cytokines involved in psoriasis pathology: anti-tumour necrosis factor (TNF), anti-interleukin (IL)-12/23, anti-IL-23, and anti-IL-17 $[1,2]$. Agents in the newer classes targeting IL17 or IL-23 show greater efficacy in phase III clinical trials than the biologics that target TNF [1, 3-6] or IL-12/23 [1, 4, 7, 8].

Treatment persistence, or drug survival, represents an important parameter related to longterm therapeutic performance in the real-life setting [9]. Biologic treatments for psoriasis are discontinued or switched in most patients, due to either to lack or loss of efficacy or tolerability issues or other complex reasons, including patient motivation [9-14]. Studies on the realworld persistence of anti-TNF and newer biologics show greater persistence for the newer agents [10-12, 15-23]. While persistence with biologics is assumed to be closely related to therapeutic performance [18], experience from other medical specialties shows that treatment patterns are influenced by many additional factors, such as patient characteristics, dosing regimens and formulations, the availability of alternative agents, and market factors, including reimbursement, pricing and marketing $[22,24,25]$.

The biologics landscape in psoriasis treatment has changed markedly in the past decade. In the European Union, four biologics targeting TNF (infliximab, etanercept, adalimumab and certolizumab pegol) were launched for this indication in 1999, 2000, 2003 and 2018, respectively, followed by patent expiry in 2015, 2015, 2018, and 2021 and the launch of biosimilars [26]. One IL-12/23 inhibitor (ustekinumab), two IL-17A inhibitors (secukinumab and ixekizumab), and one IL-17A receptor subunit blocker (brodalumab) were launched in 2009, 2015, 2016 and 2019, respectively. Three IL-23 inhibitors (guselkumab, tildrakizumab, and risankizumab) were launched in 2017, 2018 and 2019, respectively.

The impact of newly developed biologics and biosimilars on the persistence of individual agents has not been sufficiently studied. The objective of this study was to analyse the realworld persistence of biologic therapy over time in psoriasis treatment based on national registries in Sweden.

\section{METHODS}

\section{Study Design}

This retrospective, observational, longitudinal Swedish cohort study of patients used individual-level data from the Swedish National Patient Register (NPR), the Prescribed Drug Register 
(PDR) and the Cause-of-Death Register. The study was designed and implemented following the Guidelines for Good Pharmacoepidemiology Practice of the International Society for Pharmacoepidemiology [27], the STROBE (Strengthening the Reporting of Observational Studies in Epidemiology) guidelines [28] and the ethical principles specified in the Declaration of Helsinki [29]. Ethical approval was provided by the Regional Stockholm Ethics Committee (reference number 2018/1:3). Due to the non-interventional retrospective nature of the study, informed consent was not required from patients.

Included patients were adults (aged $\geq 18$ years) with a recorded diagnosis of psoriasis (International Classification of Diseases-10 (ICD-10) code L40 and subcodes) in the NPR between 1 January 2005 and 31 December 2017 , and a recorded treatment with at least one biologic in the PDR between 1 January 2010 and 31 October 2018 (Fig. 1). This study period was selected to include the widespread use of TNF inhibitors, as well as the availability of newer biologics.

Patients were excluded if treated with biologics between 2005 and 2009, treated with biologics for indications other than psoriasis, had psoriatic arthritis alone or a psoriatic arthritis diagnosis before or at the first psoriasis diagnosis or were on biologics before the first psoriasis diagnosis. These exclusion criteria ensured that only patients newly initiated on biologics to treat psoriasis were included in the analysis.

All patients meeting the inclusion and exclusion criteria were included in the study cohort. Data were linked by the National Board of Health and Welfare and merged into a single database for analyses.

\section{Biologics Included in the Study}

The biologics included in this study were three biologics that target the TNF receptor (adalimumab, infliximab and etanercept), one IL-12/ 23 inhibitor (ustekinumab), one IL-23 inhibitor (guselkumab), two IL-17A inhibitors (secukinumab and ixekizumab) and one IL-17A receptor subunit blocker (brodalumab).

Results for treatment persistence are provided for adalimumab, etanercept, secukinumab, ustekinumab and ixekizumab. Infliximab $(n=11)$, guselkumab $(n=12)$ and brodalumab $(n=4)$ were not included in the persistence analyses because of limited data, defined as $<20$ patients per treatment group.

\section{Persistence Analysis Methods}

The results of descriptive analyses for continuous variables are presented as frequencies, means, standard deviations (SD), medians and



Fig. 1 Study design. NPR Swedish National Patient Register, PsO psoriasis 
ranges (25th and 75th percentiles); those for discrete variables are presented as frequencies and percentages.

Time-to-event analysis using Kaplan-Meier methodology evaluated treatment persistence, which was defined as the time in days from the first administration to the end of drug supply following the last administration. The duration of supply of an individual administration was defined as the number of packages at the administration date multiplied by the number of defined daily doses in the package. Treatment persistence was reported as median persistence [with 95\% confidence intervals (CI)], i.e. the length of time $50 \%$ of patients remained on the specific biologic, and also as persistence rates at 1, 2 and 5 years. Treatment persistence for the 75 th percentile was also described when the median was not reached during the treatment period.

A treatment episode was defined as a treatment with a specific biologic during which patients were persistent with treatment. Patients were considered to be persistent with treatment if the gap between administrations (i.e. from the end of supply of the former administration to the administration date of the next) was less than the 'grace period' of 90 days. In accordance with previous persistence studies in psoriasis $[10,30]$, and supported by the sensitivity analyses described in the Electronic Supplementary Material files, gaps $>90$ days between administrations were assumed to be discontinuations. If patients re-initiated the same biologic after a gap of more than the 90-day grace period, they were considered to be on second-line treatment.

For the persistence analysis, only the first treatment episodes of each specific biologic treatment (i.e. adalimumab, etanercept, secukinumab, ustekinumab and ixekizumab) ever used by a patient were included in the analysis. Thus, if patients re-initiated the same biologic after the 90-day grace period, only the first treatment episode of that specific biologic was analysed. Biologic-naive treatment episodes were defined as treatment episodes in which patients had not previously received any biologic, and biologic-exposed treatment episodes were defined as treatment episodes in which patients had received a biologic before initiating the current treatment.

Descriptive analysis of change in persistence over time was also carried out for 3-year running cohorts between 2010 and 2018. Biologic treatments were grouped into treatments initiated in each calendar year from 1 January 2010 to 31 October 2018, the year before and the year after (i.e. 2010-2011, 2010-2012, 2011-2013, 2012-2014, 2013-2015, 2014-2016, 2015-2017 and 2016-2018 cohorts).

SAS version 9.4 software (SAS Institute Inc, Cary, NC, USA) was used for all data management and analysis.

\section{RESULTS}

\section{Baseline Characteristics}

In total, 178,347 patients with a diagnosis of psoriasis and/or psoriatic arthritis were identified in the NPR between 2005 and 2018. Of these, 15,738 patients had at least one administration of biologic treatment described in the PDR. Following exclusions, the primary analysis cohort comprised 2292 patients for 2010-2018 (Fig. 2).

The majority of patients (59.5\%) included in the analysis were male, ranging from $60.0 \%$ in the etanercept group to $65.3 \%$ in the adalimumab group. Mean patient age at the first psoriasis specialty visit was 42.1 years, ranging from 39.8 in the ixekizumab group to 43.3 years in the ustekinumab group. Mean time from first observable visit to specialty care of psoriasis was 7.7 years, with the shortest duration in the etanercept group (7.0 years) and longest duration in the ixekizumab group (9.1 years; Table 1).

The most common comorbidities overall were diseases of the musculoskeletal system and connective tissue [40.6\%; range 36.9\% (adalimumab group) to $43.6 \%$ (etanercept group)]; injury, poisoning and other external influences [39.3\%; range $38.0 \%$ (adalimumab group) to $43.1 \%$ (ixekizumab group)]; and diseases of the skin and subcutaneous tissue excluding psoriasis [35.5\%; range $29.4 \%$ (ixekizumab group) to $36.4 \%$ (etanercept group)]. 
PsO and/or PsA patients recorded in National Patient Registry in Sweden between 2005 and $2018(N=178,347)$

Patients treated with biologics recorded in Prescribed Drugs Registry between 2005 and $2018(N=15,738)$

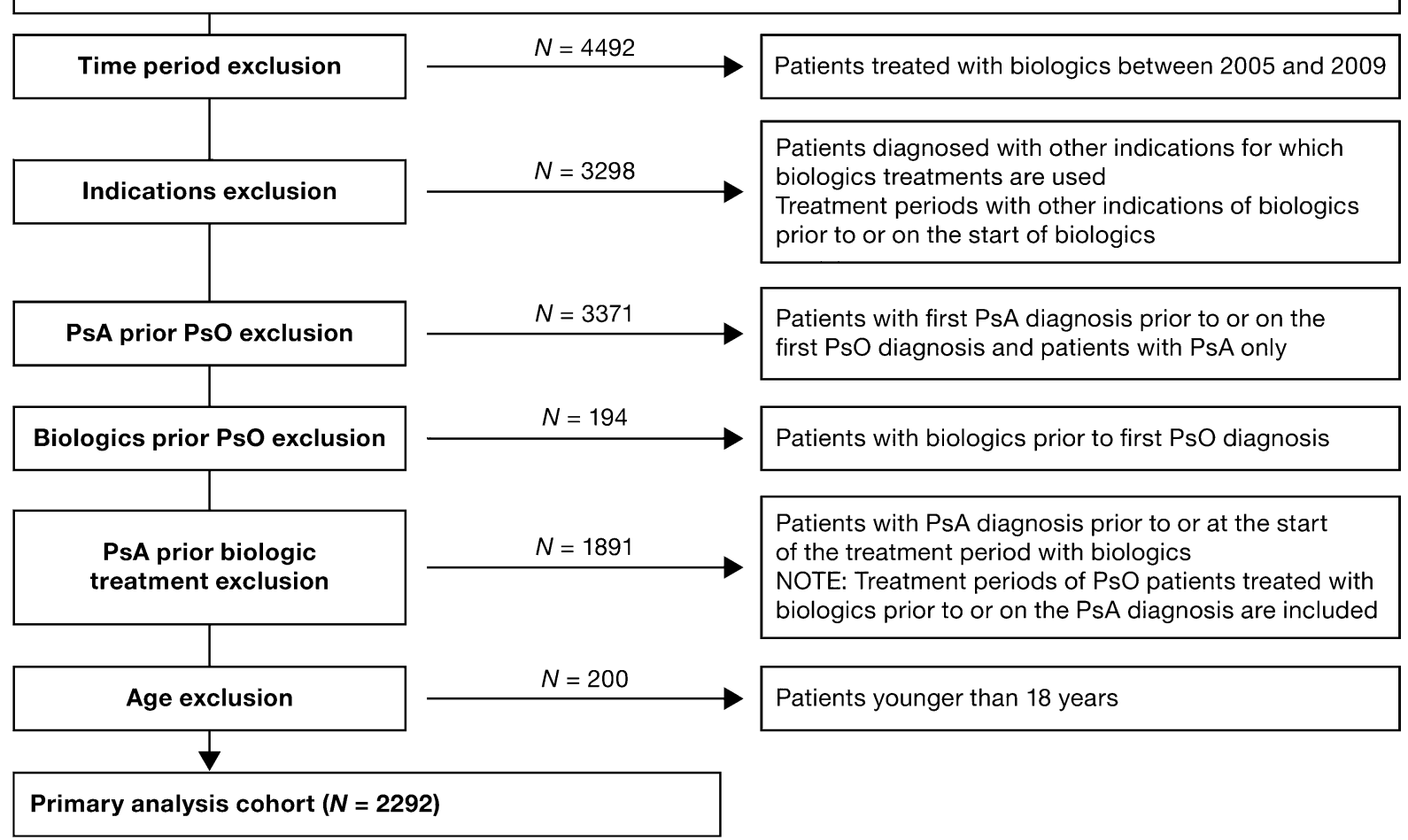

Fig. 2 STROBE diagram of the primary study population. Ps $A$ Psoriatic arthritis, STROBE Strengthening the Reporting of Observational Studies in Epidemiology

Psoriasis-related concomitant medications, including topical calcipotriol, steroids and nonbiologic systemic treatments, were prescribed for $78.7 \%$ of treatment episodes overall, most commonly in the ixekizumab (82.4\%) and etanercept (82.9\%) groups.

The mean time from psoriasis diagnosis to initiation of biologic treatment was 5.6 (SD 3.6) years, with a range from 5.3 (SD 3.4) for ustekinumab to 6.6 (SD 5.4) years for ixekizumab. In total, $75.1 \%$ of patients were biologic naive, and $24.9 \%$ were biologic exposed (Table 2). The proportions of biologic-naive patients ranged from $12.0 \%$ (ixekizumab group) to $92.8 \%$ (etanercept group).

\section{Treatment Persistence: Time-to-Event Analysis}

The overall persistence analysis comprised 3050 biologic treatment episodes, with an average of 1.33 treatment episodes per patient (all treatment episodes were included except when patients repeated a biologic they had used previously). Median persistence for all biologics overall was 23.8 months (95\% CI 21.6-26.2; Table 2). Of the treatments that reached median persistence, ustekinumab had the longest median persistence (49.3 months; 95\% CI 38.0-59.1) and etanercept the shortest (16.3 months; 95\% CI 14.5-19.0); Table 2; Fig. 3. Median persistence was not reached for ixekizumab and secukinumab, as $50 \%$ of 
Table 1 Baseline characteristics of patients treated with a biologic for psoriasis by number of treatment episodes

\begin{tabular}{|c|c|c|c|c|c|c|}
\hline Baseline characteristics & Overall $^{\mathbf{a}}$ & Adalimumab & Etanercept & Ixekizumab & Secukinumab & Ustekinumab \\
\hline $\begin{array}{l}\text { Number of treatment } \\
\text { episodes, } n\end{array}$ & 3747 & 1448 & 1125 & 51 & 441 & 564 \\
\hline $\begin{array}{l}\text { Number of patients } \\
\text { with } \geq 1 \text { treatment } \\
\text { episode, } n\end{array}$ & 2292 & 1046 & 974 & 50 & 394 & 488 \\
\hline Male, $n(\%)$ & $2230(59.5)$ & $946(65.3)$ & $675(60.0)$ & $31(60.8)$ & $267(60.5)$ & $354(62.8)$ \\
\hline \multicolumn{7}{|l|}{ Age at diagnosis } \\
\hline Mean (SD) & $42.1(14.2)$ & $41.1(13.9)$ & $42.6(14.4)$ & $39.8(14.4)$ & $42.6(14.0)$ & $43.3(14.4)$ \\
\hline Median (Q1, Q3) & $41(31,54)$ & $40(30,51)$ & $42(31,54)$ & $41(26,53)$ & $42(31,55)$ & $43(32,55)$ \\
\hline Minimum, maximum & 18,83 & 18,82 & 18,83 & 18,64 & 18,76 & 18,83 \\
\hline $\begin{array}{l}\text { PsA diagnosis after index } \\
\text { treatment, } n(\%)\end{array}$ & $287(7.7)$ & $115(7.9)$ & $110(9.8)$ & 0 & $12(2.7)$ & $34(6.0)$ \\
\hline \multicolumn{7}{|c|}{ Psoriasis-related concomitant medication, $n$ (\%) } \\
\hline Overall & $2950(78.7)$ & $1123(77.6)$ & $933(82.9)$ & $42(82.4)$ & $344(78.0)$ & $446(79.1)$ \\
\hline Topical calcipotriol & $2067(55.2)$ & $646(44.6)$ & $547(48.6)$ & $23(45.1)$ & $185(42.0)$ & $256(45.4)$ \\
\hline Topical steroids & $1726(46.1)$ & $752(51.9)$ & $610(54.2)$ & $26(51.0)$ & $257(58.3)$ & $341(60.5)$ \\
\hline $\begin{array}{l}\text { Non-biologic systemic } \\
\text { treatments }\end{array}$ & $817(21.8)$ & $342(23.7)$ & $294(26.1)$ & $4(7.8)$ & $64(14.5)$ & $73(12.9)$ \\
\hline \multicolumn{7}{|c|}{ Medications dispensed during biologic treatment episodes, $n$ (\%) } \\
\hline Topical calcipotriol & $1329(35.5)$ & $508(35.1)$ & $435(38.7)$ & $15(29.4)$ & $142(32.2)$ & $210(37.2)$ \\
\hline Topical steroids & $1664(44.4)$ & $624(43.1)$ & $502(44.6)$ & $23(45.1)$ & $210(47.6)$ & $277(49.1)$ \\
\hline Systemic treatments & $485(12.9)$ & $225(15.5)$ & $175(15.6)$ & $3(5.9)$ & $24(5.4)$ & $27(4.8)$ \\
\hline $\begin{array}{l}\text { Immunosuppressive } \\
\text { treatments }\end{array}$ & $16(0.4)$ & $6(0.4)$ & $5(0.4)$ & 0 & $1(0.2)$ & $4(0.7)$ \\
\hline \multicolumn{7}{|c|}{ Previous biologic treatments, $n(\%)^{c}$} \\
\hline 0 & $2292(61.2)$ & $862(59.5)$ & $904(80.4)$ & $6(11.8)$ & $202(45.8)$ & $254(45.0)$ \\
\hline 1 & $874(23.3)$ & $274(18.9)$ & $121(10.8)$ & $38(74.5)$ & $179(40.6)$ & $224(39.7)$ \\
\hline 2 & $341(9.1)$ & $163(11.3)$ & $60(5.3)$ & $5(9.8)$ & $53(12.2)$ & $53(9.4)$ \\
\hline 3 & $136(3.6)$ & $78(5.4)$ & $25(2.2)$ & $2(3.9)$ & $6(1.4)$ & $21(3.7)$ \\
\hline$\geq 4$ & $104(2.8)$ & $71(4.9)$ & $15(1.3)$ & 0 & $1(0.2)$ & $12(2.3)$ \\
\hline \multicolumn{7}{|c|}{ Duration of $\mathrm{PsO}$ (from the first $\mathrm{PsO}$ diagnosis to end of the follow-up), years } \\
\hline Mean (SD) & $7.7(3.7)$ & $7.9(3.7)$ & $7.0(4.5)$ & $9.1(3.5)$ & $7.8(3.8)$ & $8.5(3.5)$ \\
\hline Median (Q1, Q3) & $7.4(4.7,10.8)$ & $\begin{array}{c}7.9(4.9 \\
11.0)\end{array}$ & $\begin{array}{c}6.7(4.0 \\
10.0)\end{array}$ & $\begin{array}{c}9.3(5.9 \\
13.0)\end{array}$ & $7.4(5.0,10.2)$ & $8.5(5.7,11.4)$ \\
\hline
\end{tabular}


Table 1 continued

\begin{tabular}{lllllll}
\hline Baseline characteristics & Overall $^{\text {a }}$ & Adalimumab & Etanercept & Ixekizumab & Secukinumab & Ustekinumab \\
\hline $\begin{array}{c}\text { Minimum, maximum } \\
\text { Hospitalization for PsO }\end{array}$ & $0.1,14.6$ & $0.2,14.0$ & $0.1,14.0$ & $1.0,14.1$ & $0.7,14.3$ & $0.4,14.6$ \\
$\begin{array}{l}1 \text { year prior to initiation } \\
\text { of biologic, } n \text { (\%) }\end{array}$ & $104(2.8)$ & $36(2.5)$ & $33(2.9)$ & 0 & $14(3.2)$ & $20(3.6)$ \\
$\begin{array}{l}\text { Outpatient visits 1 year } \\
\text { prior initiation of } \\
\text { biologic, } n \text { (\%) }\end{array}$ & $2885(77.0)$ & $1157(79.9)$ & $860(76.4)$ & $37(72.6)$ & $329(74.6)$ & $468(83.0)$ \\
\hline
\end{tabular}

$P s A$ Psoriatic arthritis, $P s O$ psoriasis, $Q 125$ th percentile, $Q 375$ th percentile, $S D$ standard deviation

${ }^{a}$ Numbers of patients treated with specific biologics will not sum up to the overall number of patients $(N=2292)$ as patients could use several different biologic treatments throughout their treatment course; data are not shown for biologics with $n<50$ (48 patients were treated with golimumab, 23 were treated with certolizumab pegol, 12 were treated with guselkumab, 11 were treated with infliximab and 4 were treated with brodalumab); however, they were included in the overall group.

b Defined as methotrexate, apremilast and immunosuppressives

${ }^{c}$ Patients who were treated with biologic therapy before 2010 were excluded. Patients might have had more than 1 treatment episode of biologics. If the patient had 2 treatment episodes with biologics, the second treatment episode was regarded as biologic exposure and, therefore, marked as having 1 previous biologic treatment episode

${ }^{d} \mathrm{PsO}$ as primary diagnosis

patients did not discontinue treatment within the time frame of the study.

When treatments were divided according to biologic-naive and biologic-exposed treatment episodes, patients receiving ustekinumab were the most persistent in both the biologic-naive (55.4 months; 95\% CI 45.6-64.8) and biologicexposed (40.3 months; 95\% CI 32.6-58.7) groups, with a greater persistence with the former (when considering treatments that reached median persistence). The biologics with the least persistence in the biologic-naive group

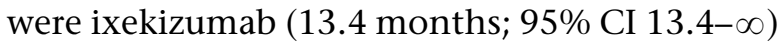
and etanercept (16.7 months; 95\% CI 15.0-19.8). Median persistence was not reached for biologic-exposed patients treated with ixekizumab and biologic-naive patients treated with secukinumab.

Persistence rates in the overall cohort at 1 year from treatment initiation were highest for the ixekizumab (81.3\%) and ustekinumab (79.9\%) and lowest for etanercept (57.8\%) and adalimumab (64.6\%), with secukinumab intermediate (75.9\%). Persistence at 2 years remained highest for ustekinumab (64.8\%), with lower rates for secukinumab (58.5\%), adalimumab (47.9\%) and etanercept (39.7\%). Similarly, persistence at 5 years was highest for ustekinumab (41.6\%), with lower persistence for adalimumab (26.8\%) and etanercept $(16.8 \%)$. Results for secukinumab at 5 years and ixekizumab at 2 and 5 years were not available as these biologics had not been on the market for these durations.

In the biologic-naive subgroup, patients receiving ustekinumab were most persistent at 1,2 and 5 years $(82.6,66.8$ and $46.6 \%$, respectively), and those receiving etanercept patients were least persistent (59.1, 40.8 and $17.3 \%$, respectively). In the biologic-exposed subgroup the pattern was similar, with the highest proportion of patients still on treatment in the ixekizumab (78.4\% at 1 year) and ustekinumab groups $(76.7 \%$ at 1 year, $62.6 \%$ at 2 years, $33.0 \%$ at 5 years) and the lowest proportion still on treatment for etanercept (42.6, 25.3 and 10.1\%, respectively). Adalimumab and secukinumab 


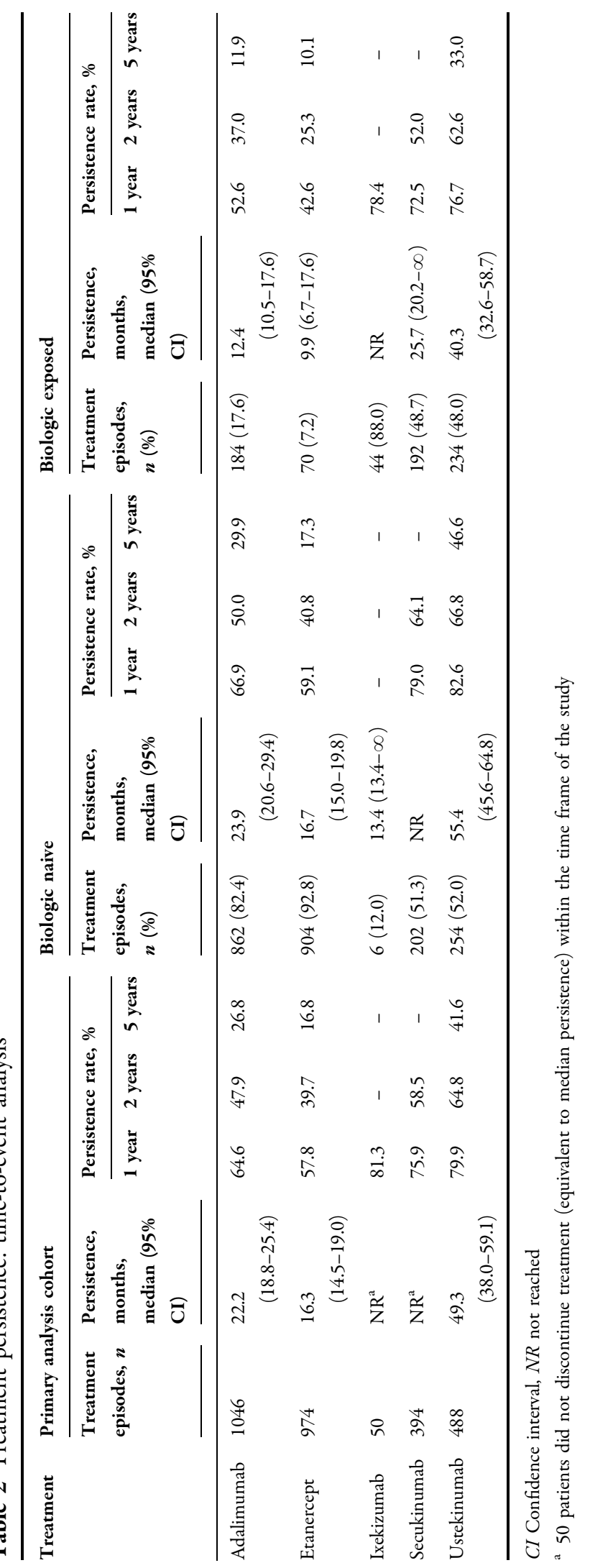






Fig. 3 Kaplan-Meier plot of persistence by biologic treatment time-to-event analysis

showed intermediate persistence in both the biologic-naive and biologic-exposed subgroups.

\section{Treatment Persistence Over Time in 3-Year Running Cohorts: Time-to-Event Analysis}

Persistence for biologics was grouped into 3 -year running cohorts between 1 January 2010 and 31 October 2018 (Table 3; Fig. 4). Median persistence for ustekinumab decreased by almost 50\% between 2010-2011 and

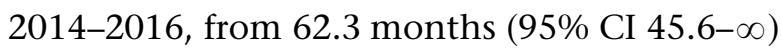
to 32.7 months (21.2-49.3; Table 3 ) and did not reach a median persistence during 2015-2017 and 2016-2018. For secukinumab, the median persistence was not reached, except in

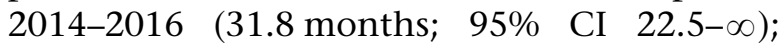
persistence at the 75 th percentile was longest in 2013-2015, at 21.5 months (95\% CI 10.4-31.8) and showed substantial decreases in subsequent years.

Both adalimumab and etanercept showed relatively stable persistence over time, reaching longest persistence in 2016-2018 (median 23.5 months, $95 \%$ CI $18.7-\infty$ and 18.7 months,

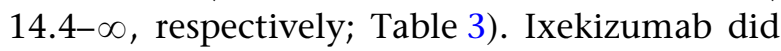
not reach a median persistence for any 3-year cohort and reached the 75th percentile threshold only in the cohort initiated in 2016-2018; therefore, no trend over time could be observed.

\section{DISCUSSION}

In this retrospective, observational registry study in Sweden we assessed treatment persistence and change in persistence over time for individual biologics used in the treatment of psoriasis. The patients assessed had broadly similar demographic and clinical characteristics across the biologic groups analysed.

Overall, the median persistence for biologics was approximately 2 years. Because psoriasis is a chronic disease that usually requires lifelong treatment, this level of persistence can be considered low. Greater persistence was seen for ustekinumab (IL-12/23 inhibitor) and secukinumab (IL-17A inhibitor) than for the anti-TNF biologics etanercept and adalimumab. Our observations on the persistence of ustekinumab are consistent with those from previous realworld studies, which also reported greater persistence with ustekinumab than with TNF biologics $[9,11,23,31]$. For secukinumab, our 


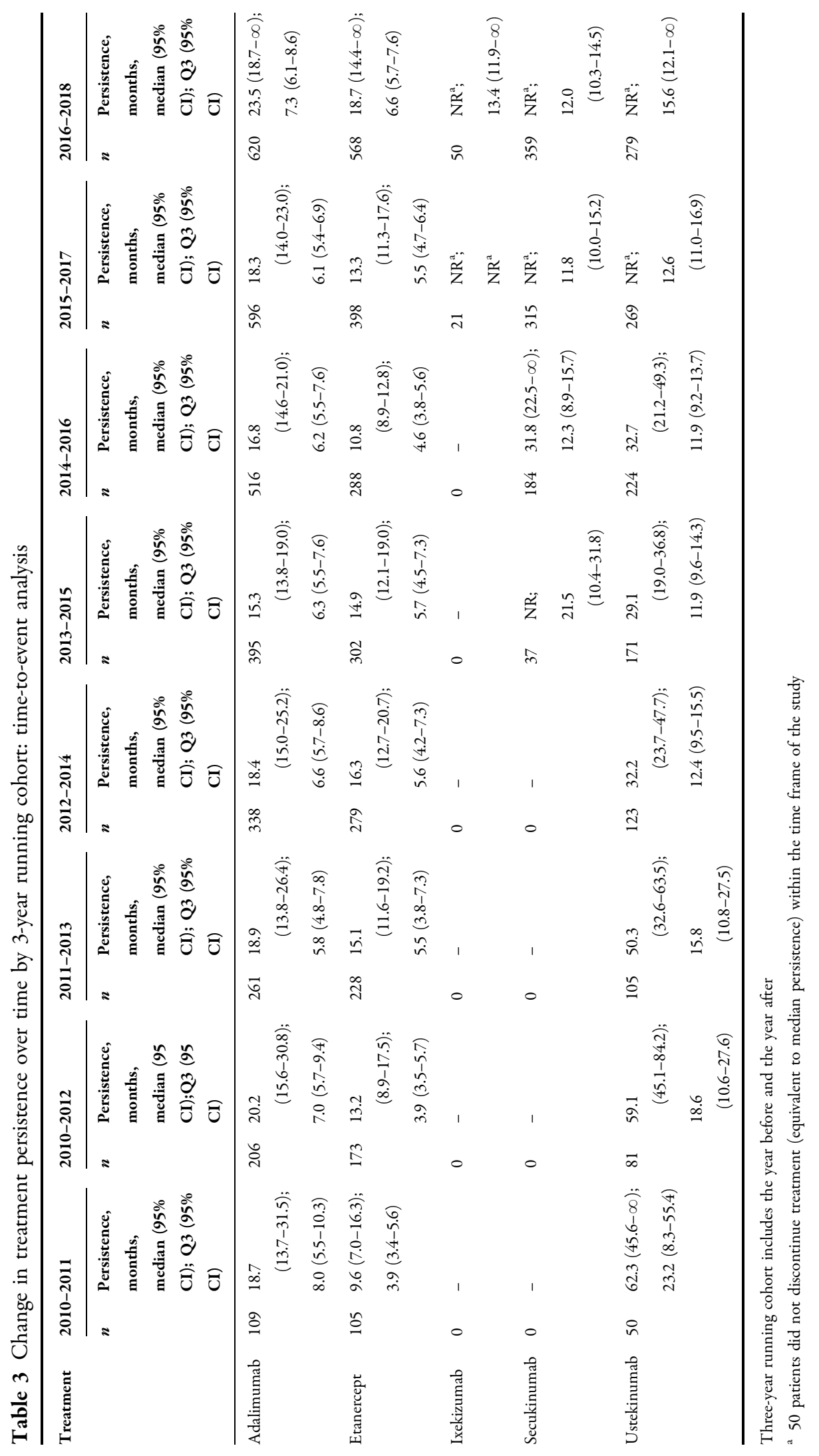



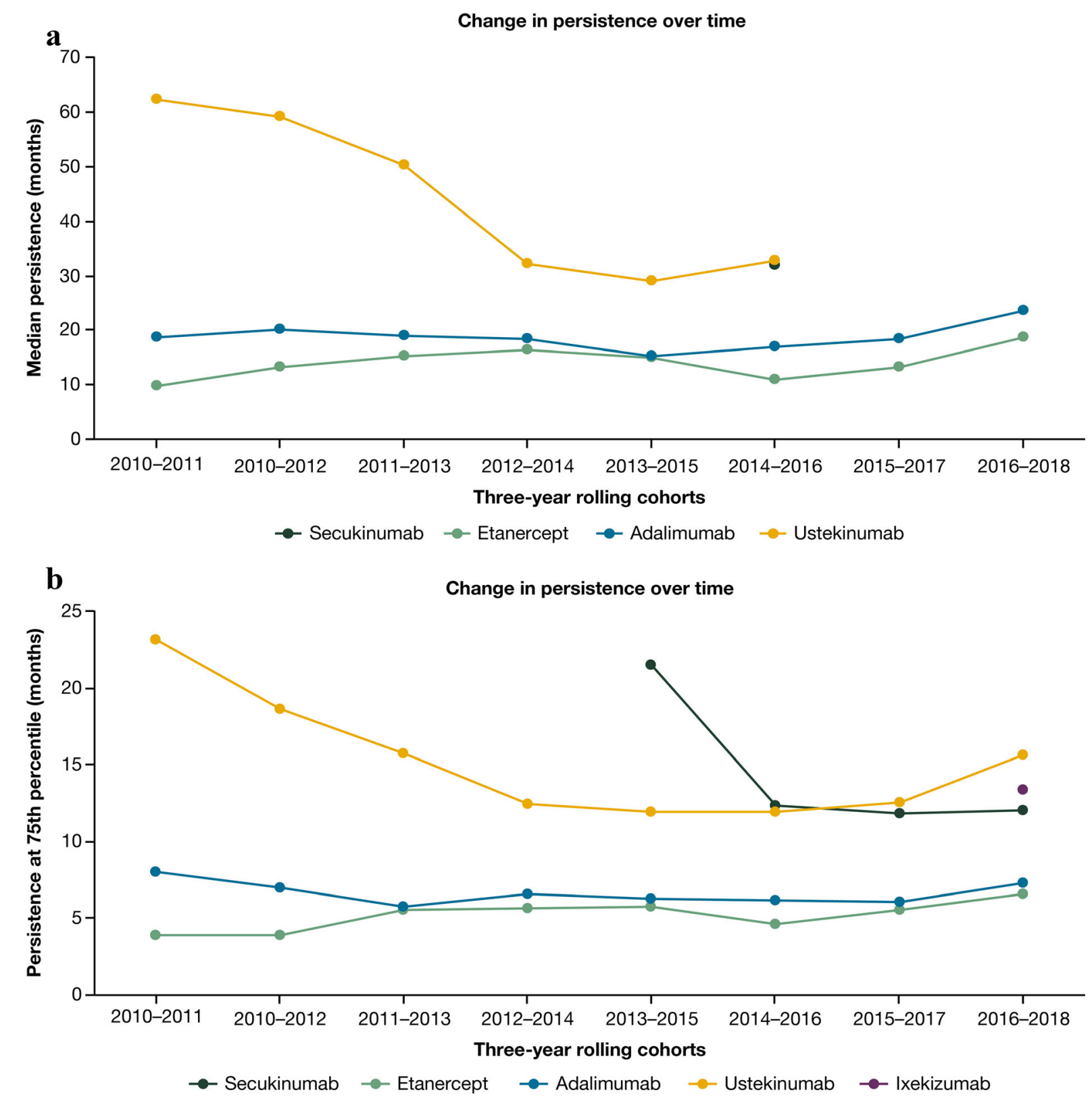

Fig. 4 Change in median (a) and 75th percentile (b) persistence over time from 2010 to 2018 by 3-year rolling cohort $^{\mathrm{a}}$ : time-to-event analysis. ${ }^{a}$ Three-year rolling cohort includes the year before and the year after initiation of treatment

results contrast with those of earlier studies reporting low persistence $[11,16]$, although these reports were potentially influenced by the high proportion of biologic-experienced patients who were treated with secukinumab [10]. Our observations on the IL-17A inhibitor ixekizumab indicated low levels of persistence comparable to that with etanercept. However, these observations were based on very few patients with limited time in the database; therefore, more data are needed to draw conclusions on its persistence. Other real-world 
studies have reported greater persistence for ixekizumab than for adalimumab or secukinumab [17, 18].

For each of the biologics analysed, median persistence was greater in biologic-naive than biologic-exposed patients, in agreement with findings from previous studies $[9,10,15,32,33]$. One explanation for the reduction in persistence in biologic-exposed patients is that patients requiring second-line treatment might have more refractory disease [22]. Specifically, in relation to biologic-naive patients in our analysis, persistence was higher for ustekinumab and secukinumab, and lower for adalimumab and etanercept, consistent with our overall findings. Analysis of the biologicnaive ixekizumab group included only six patients, which influences the robustness of these outcomes.

Kaplan-Meier analysis showed that persistence at 1,2 , and 5 years ranged from highest rates for ustekinumab $(79.9,64.8,41.6 \%$, respectively) to the lowest rates for etanercept $(57.8,39.7,16.8 \%)$. These rates confirm that patients are likely to receive multiple different treatments in the course of their disease and underline the importance of selecting the right treatment options at an early stage.

In the 3-year running cohorts between 2010 and 2018, we observed a notable, progressive decrease in the median persistence for ustekinumab. This decrease, starting with the 2011-2013 cohort [ending persistence in 2015-2017 (median 50.3 months later)] and decreasing even more in the 2012-2014 cohort [ending persistence in 2015-2017 (median 32.2 months later)], coincided with the entry of newer biologics and the availability of biosimilars. It might be speculated that the availability of new biologics for patients who had run out of treatment options facilitated switching in this period. For etanercept, persistence initially increased for the 2012-2014 cohort (ending persistence 13.2 months later), then decreased and then increased again for the 2016-2018 cohort to approximately double the lowest level observed, potentially reflecting changes in the cost of this biologic at the introduction of biosimilars and confidential side agreements and discounts. Physicians continued to use adalimumab in a relatively stable way throughout the study period. It can be speculated that persistence was higher for newer drugs during initial treatment due to launch excitement and the close monitoring of patients with a high level of patience by clinicians. Complex interactions, including perceptions of relative therapeutic performance and market dynamics, are likely to play a part in changing persistence over time for each biologic.

The primary strength of this study is that it analysed the entire Swedish population using prescription data rich in detail and prescriptions linked with diagnoses at the individual level, enabling an accurate selection of patients with psoriasis. The limitations of real-world studies also apply to our analyses. As this was a retrospective observational study, the data were not specifically collected for the purpose of the study, treatment groups were not matched for patient characteristics or previous biologic treatment history and the duration of treatment differed among biologics. It was not possible to assess the reasons for initiating and discontinuing individual biologics and the role of patient characteristics or market factors, such as reimbursement decisions and price negotiations, in determining persistence. Together with persistence, adherence to therapy is important for optimizing patient care and therapeutic outcomes. However, analysis of adherence to the dosing scheme was not possible in this study, as information on the dose taken was not available in the national patient registries. It was assumed that all dispensed drugs were taken and that the date of dispensation was also a date of treatment use. Additionally, our study included a 90-day grace period to account for any gaps in adherence. Differences among biologics were not statistically evaluated, although the trends observed offer robust insights into real-world treatment patterns for biologics to treat psoriasis in Sweden. Finally, although we used a 90-day grace period as in previous literature and evaluated the level through sensitivity analyses, there are still patients who likely did not discontinue but rather had a long 'drug holiday.'

Our results suggest that the clinical realworld persistence for interleukin inhibitors might not be as long as reported previously, 
which is a problem because treatment persistence has often been associated with treatment success. Our findings suggest that clinicians might have kept patients on these biologics because they were the best choices available at a given time. With newly available treatment alternatives, more recent data on persistence will provide a better estimate of treatment success in psoriasis.

Specifically, this analysis suggests that the persistence for ustekinumab has decreased over time, which might be explained by the introduction of more biologic treatment options from 2015 forward. Hence, the greater overall persistence for ustekinumab might be explained by the fact that ustekinumab was the only interleukin inhibitor on the market between 2009 and 2015 in Sweden.

\section{CONCLUSIONS}

This analysis of Swedish registry data shows that the persistence of biologic therapy for psoriasis is low on average (approximately 2 years) given that psoriasis can be considered to be a chronic disease that usually requires lifelong treatment. Our findings suggest that overall persistence is greater for newer interleukin inhibitor biologics than for TNF inhibitors. Thereby, this study contributes to the body of evidence on the overall persistence for biologics with important evidence on the differential persistence over time for each biologic.

These results indicate that persistence has changed over time for some biologics and that future studies on persistence should include analyses of persistence over time to provide an accurate picture of the current treatment landscape. This may help clinicians to make informed decisions when choosing a biologic treatment for their patients with psoriasis.

\section{ACKNOWLEDGEMENTS}

Funding. The study, with the exception of the contributions of MS-E. and L-ÅL, was funded by LEO Pharma A/S. LEO Pharma employees contributed to the interpretation of data and participated in the review and approval of the publication. The sponsor also funded the journal's Rapid Service fee.

Medical Writing and Editorial Assistance. Editorial assistance was provided by Breanne Landry at Parexel, with funding from LEO Pharma A/S.

Authorship. All named authors meet the International Committee of Medical Journal Editors (ICMJE) criteria for authorship of this article, take responsibility for the integrity of the work as a whole, and have given their approval for this version to be published.

Authors' Contributions. M Schmitt-Egenolf contributed to the conceptualization, methodology and supervision of the study, validated the data and critically reviewed the manuscript. J. Freilich contributed to the conceptualization, data curation, investigation, methodology, project administration, resources and supervision of the study, validated the data and visualized and critically reviewed the manuscript. NM Stelmaszuk-Zadykowicz contributed to the data curation, formal analysis, investigation, methodology, resources and software of the study, validated the data and visualized and critically reviewed the manuscript. E Apol contributed to the conceptualization, funding acquisition, investigation, methodology, project administration and supervision of the study, validated the data and visualized and critically reviewed the manuscript. JB Hansen contributed to the conceptualization, funding acquisition, investigation, methodology and supervision of the study, validated the data and visualized and critically reviewed the manuscript. L-Å Levin contributed to the conceptualization, methodology and supervision of the study, validated the data and critically reviewed the manuscript. All authors approved the final manuscript as submitted.

Disclosures. Marcus Schmitt-Egenolf is responsible for dermatology in project management for the National Psoriasis Guidelines at the Swedish Board of Health and Welfare. 
Jonatan Freilich and Natalia M. StelmaszukZadykowicz are employees of Parexel International. Eydna Apol and Jes B. Hansen are employees of LEO Pharma A/S. Lars-Åke Levin has received consulting fees from LEO Pharma, Biogen, Novartis, AbbVie, Galderma, UCB and Pfizer.

Compliance with Ethical Guidelines. The study was designed and implemented following the Guidelines for Good Pharmacoepidemiology Practice of the International Society for Pharmacoepidemiology, the STROBE (Strengthening the Reporting of Observational Studies in Epidemiology) guidelines, and the ethical principles specified in the Declaration of Helsinki. Ethical approval was provided by the Regional Stockholm Ethics Committee (reference number 2018/1:3). Due to the non-interventional retrospective nature of the study, informed consent was not required from patients.

Data Availability. The datasets generated during and/or analysed during the current study are available from the corresponding author on reasonable request.

Open Access. This article is licensed under a Creative Commons Attribution-NonCommercial 4.0 International License, which permits any non-commercial use, sharing, adaptation, distribution and reproduction in any medium or format, as long as you give appropriate credit to the original author(s) and the source, provide a link to the Creative Commons licence, and indicate if changes were made. The images or other third party material in this article are included in the article's Creative Commons licence, unless indicated otherwise in a credit line to the material. If material is not included in the article's Creative Commons licence and your intended use is not permitted by statutory regulation or exceeds the permitted use, you will need to obtain permission directly from the copyright holder. To view a copy of this licence, visit http:// creativecommons.org/licenses/by-nc/4.0/.

\section{REFERENCES}

1. Sbidian E, Chaimani A, Afach S, et al. Systemic pharmacological treatments for chronic plaque psoriasis: a network meta-analysis. Cochrane Database Syst Rev. 2020;1(1):Cd011535.

2. Girolomoni G, Strohal R, Puig L, et al. The role of IL-23 and the IL-23/TH 17 immune axis in the pathogenesis and treatment of psoriasis. J Eur Acad Dermatol Venereol. 2017;31(10):1616-26.

3. Gordon KB, Blauvelt A, Papp KA, et al. Phase 3 trials of ixekizumab in moderate-to-severe plaque psoriasis. N Engl J Med. 2016;375(4):345-56.

4. Langley RG, Armstrong AW, Lebwohl MG, et al. Efficacy and safety of brodalumab in patients with psoriasis who had inadequate responses to ustekinumab: subgroup analysis of two randomized phase III trials. Br J Dermatol. 2019;180(2):306-14.

5. Blauvelt A, Papp KA, Griffiths CE, et al. Efficacy and safety of guselkumab, an anti-interleukin-23 monoclonal antibody, compared with adalimumab for the continuous treatment of patients with moderate to severe psoriasis: results from the phase III, double-blinded, placebo- and active comparatorcontrolled VOYAGE 1 trial. J Am Acad Dermatol. 2017;76(3):405-17.

6. Griffiths CE, Reich K, Lebwohl M, et al. Comparison of ixekizumab with etanercept or placebo in moderate-to-severe psoriasis (UNCOVER-2 and UNCOVER-3): results from two phase 3 randomised trials. Lancet. 2015;386(9993):541-51.

7. Reich K, Pinter A, Lacour JP, et al. Comparison of ixekizumab with ustekinumab in moderate-to-severe psoriasis: 24-week results from IXORA-S, a phase III study. Br J Dermatol. 2017;177(4):1014-23.

8. Lebwohl M, Strober B, Menter A, et al. Phase 3 studies comparing brodalumab with ustekinumab in psoriasis. N Engl J Med. 2015;373(14):1318-28.

9. Gniadecki R, Bang B, Bryld LE, et al. Comparison of long-term drug survival and safety of biologic agents in patients with psoriasis vulgaris. Br J Dermatol. 2015;172(1):244-52.

10. Yiu ZZN, Mason KJ, Hampton PJ, et al. Drug survival of adalimumab, ustekinumab and secukinumab in patients with psoriasis: a prospective cohort study from the British Association of Dermatologists Biologics and Immunomodulators Register (BADBIR). Br J Dermatol. 2020;183(2): 294-302.

11. Egeberg A, Ottosen MB, Gniadecki R, et al. Safety, efficacy and drug survival of biologics and 
biosimilars for moderate-to-severe plaque psoriasis. Br J Dermatol. 2018;178(2):509-19.

12. No DJ, Inkeles MS, Amin M, et al. Drug survival of biologic treatments in psoriasis: a systematic review. J Dermatolog Treat. 2018;29(5):460-6.

13. Belinchon I, Rivera R, Blanch C, et al. Adherence, satisfaction and preferences for treatment in patients with psoriasis in the European Union: a systematic review of the literature. Patient Prefer Adherence. 2016;10:2357-67.

14. Levin EC, Gupta R, Brown $G$, et al. Biologic fatigue in psoriasis. J Dermatolog Treat. 2014;25(1):78-82.

15. Egeberg A, Bryld LE, Skov L. Drug survival of secukinumab and ixekizumab for moderate-to-severe plaque psoriasis. J Am Acad Dermatol. 2019;81(1):173-8.

16. van den Reek J, van Vugt LJ, van Doorn MBA, et al. Initial results of secukinumab drug survival in patients with psoriasis: a multicentre daily practice cohort study. Acta Derm Venereol. 2018;98(7): 648-54.

17. Blauvelt A, Shi N, Burge R, et al. Comparison of realworld treatment patterns among patients with psoriasis prescribed ixekizumab or secukinumab. J Am Acad Dermatol. 2020;82(4):927-35.

18. Blauvelt A, Shi N, Burge R, et al. Comparison of realworld treatment patterns among psoriasis patients treated with ixekizumab or adalimumab. Patient Prefer Adherence. 2020;14:517-27.

19. Marinas JE, Kim WB, Shahbaz A, et al. Survival rates of biological therapies for psoriasis treatment in real-world clinical practice: a Canadian multicentre retrospective study. Australas J Dermatol. 2018;59(1):e11-4.

20. Roche H, Bouiller K, Puzenat E, et al. Efficacy and survival of biologic agents in psoriasis: a practical real-life 12-year experience in a French dermatology department. J Dermatolog Treat. 2019;30(6):540-4.

21. Carter C, Wilson KL, Smith D, et al. Comparative treatment patterns among psoriasis patients using adalimumab, etanercept, or ustekinumab. Am J Pharm Benefits. 2016;8(5):191-8.

22. Menter A, Papp KA, Gooderham M, et al. Drug survival of biologic therapy in a large, disease-based registry of patients with psoriasis: results from the Psoriasis Longitudinal Assessment and Registry (PSOLAR). J Eur Acad Dermatol Venereol. 2016;30(7):1148-58.

23. Lin PT, Wang SH, Chi CC. Drug survival of biologics in treating psoriasis: a meta-analysis of realworld evidence. Sci Rep. 2018;8(1):16068.
24. Pettersson B, Hoffmann M, Andersson D, et al. Utilization and costs of glucose lowering therapies following health technology assessment for the new reimbursement scheme in Sweden. Health Policy. 2012;108(2-3):207-15.

25. Pettersson B, Hoffmann $M$, Wändell $P$, et al. Utilization and costs of lipid modifying therapies following health technology assessment for the new reimbursement scheme in Sweden. Health Policy. 2012;104(1):84-91.

26. Generics and Biosimilars Initiative. Biologicals patent expiries. 2015. http://www.gabionline.net/ Biosimilars/General/Biologicals-patent-expiries. Accessed 30 Sept 2021.

27. Epstein M. Guidelines for good pharmacoepidemiology practices (GPP). Pharmacoepidemiol Drug Saf. 2005;14(8):589-95.

28. Vandenbroucke JP, von Elm E, Altman DG, et al. Strengthening the Reporting of Observational Studies in Epidemiology (STROBE): explanation and elaboration. PLoS Med. 2007;4(10):e297.

29. World Medical Association. WMA Declaration of Helsinki-ethical principles for medical research involving human subjects. 2018. https://www. wma.net/policies-post/wma-declaration-ofhelsinki-ethical-principles-for-medical-researchinvolving-human-subjects/. Accessed 30 Sept 2021.

30. Warren RB, Smith CH, Yiu ZZN, et al. Differential drug survival of biologic therapies for the treatment of psoriasis: a prospective observational cohort study from the British Association of Dermatologists Biologic Interventions Register (BADBIR). J Invest Dermatol. 2015;135(11):2632-40.

31. Sbidian E, Mezzarobba M, Weill A, et al. Persistence of treatment with biologics for patients with psoriasis: a real-world analysis of 16,545 biologic-naive patients from the French National Health Insurance database (SNIIRAM). Br J Dermatol. 2019;180(1): 86-93.

32. Iskandar IYK, Warren RB, Lunt M, et al. Differential drug survival of second-line biologic therapies in patients with psoriasis: observational cohort study from the British Association of Dermatologists Biologic Interventions Register (BADBIR). J Invest Dermatol. 2018;138(4):775-84.

33. van den Reek JM, Zweegers J, Kievit W, et al. "Happy" drug survival of adalimumab, etanercept and ustekinumab in psoriasis in daily practice care: results from the BioCAPTURE network. Br J Dermatol. 2014;171(5):1189-96. 